\title{
Quantitation of Overlapping Core Edges in EFTEM Spectrum Imaging of Cells
}

\author{
R.D. Leapman and M.A. Aronova
}

Laboratory of Cellular Imaging \& Macromolecular Biophysics, NIBIB, NIH, Bethesda, MD 20892

In applications of analytical electron microscopy to cell biology, it is often necessary to study compositional variations over $10-\mu \mathrm{m}$ sized areas encompassing entire sections through cells, and furthermore to perform such measurements on many cells under different experimental conditions [1]. Although spectrum-imaging in the scanning transmission electron microscope (STEM) provides the most complete electron energy loss spectroscopic (EELS) data at each pixel in a compositional map [2,3], STEM-EELS is often limited in terms of the maximum probe current (e.g., $1 \mathrm{nA}$ ), and acquisition speed (e.g., $5 \mathrm{~ms}$ per spectrum). Despite the lower efficiency of EFTEM spectrumimaging, for which spectral information must be acquired sequentially one energy loss at a time, much higher total currents $(\sim 100 \mathrm{nA})$ are available with wide-beam illumination, and large numbers of pixels $\left(\sim 10^{6}\right)$ can be read out in a few seconds [4].

The EFTEM spectrum-imaging approach can offer advantages relative to STEM-EELS for applications of elemental mapping when radiation damage is not a limiting factor, and elements of interest are present at relatively high concentrations. In particular, the approach can be used to map distributions of nucleic acids based on the phosphorus signal ( $\mathrm{P} \mathrm{L}_{2,3}$ edge at $132 \mathrm{eV}$ ) and distributions of proteins that are rich in the sulfur-containing amino acids cysteine and methionine, based on the sulfur signal ( $\mathrm{S} \mathrm{L}_{2,3}$ edge at $160 \mathrm{eV}$ ) [5]. However, quantitative analysis of these elements is complicated by the strong overlap of their $\mathrm{L}_{2,3}$ edges. Here we show that it is possible to perform multiple least squares fitting of EFTEM spectrum-image data to separate the distributions of phosphorus and sulfur in unstained sections of embedded tissues and cells.

Fig. 1 shows elemental distributions obtained by EFTEM spectrum-imaging of a beta cell in a thin section of epon-embedded mouse pancreatic islet [6]. The phosphorus and sulfur images were derived from a multiple least squares (MLS) fit of experimentally determined $\mathrm{P}$ and $\mathrm{S} \mathrm{L}_{2,3}$ reference spectra (shown in the lower right), and a single-scattering background spectrum modeled to an inverse power law, as well as a double scattering reference spectrum obtained by convolving the measured background with the measured low-loss spectrum. Since the specimen contained some silicon contamination, the $\mathrm{Si} \mathrm{L}_{2,3}$ edge was also included in the MLS fit. Taken together these reference spectra resulted in a good quality of fit. As evident in Fig. 1, high levels of sulfur are found in the storage granules containing insulin, whose amino acid sequence has $12 \%$ cysteine residues. No phosphorus is observed in the storage granules but phosphorus is concentrated in the chromatin of the nucleus as well as in ribosomes in the cytoplasm. As expected, nitrogen is seen throughout the cell in both the nucleus and in the storage granules.

We are currently applying EFTEM spectrum-imaging together with MLS fitting of reference spectra to determine the insulin content of beta cell granules in pancreatic islets of Langerhans; to map distributions of proteins and nucleic acids in cell nuclei to study supramolecular complexes involved in gene regulation; and to determine distributions of organic components in hybrid nanoparticles used in medical imaging [7]. 


\section{References}

[1] M.A. Aronova, R.D. Leapman, MRS Bulletin 37 (2012) 53.

[2] H. Shuman, A.P. Somlyo, Ultramicroscopy 21 (1987) 23.

[3] R.D. Leapman, J. Microsc. 210 (2003) 5.

[4] G. Goping et al., Microsc. Res. Tech. 61 (2003) 448.

[5] R.D. Leapman, M. Jarnik, A.C. Steven, J. Struct. Biol. 120 (1997) 168.

[6] T. Cai et al., Diabetologia 54 (2011) 2347.

[7] This research was supported by the intramural program of the National Institute of Biomedical Imaging and Bioengineering, NIH.
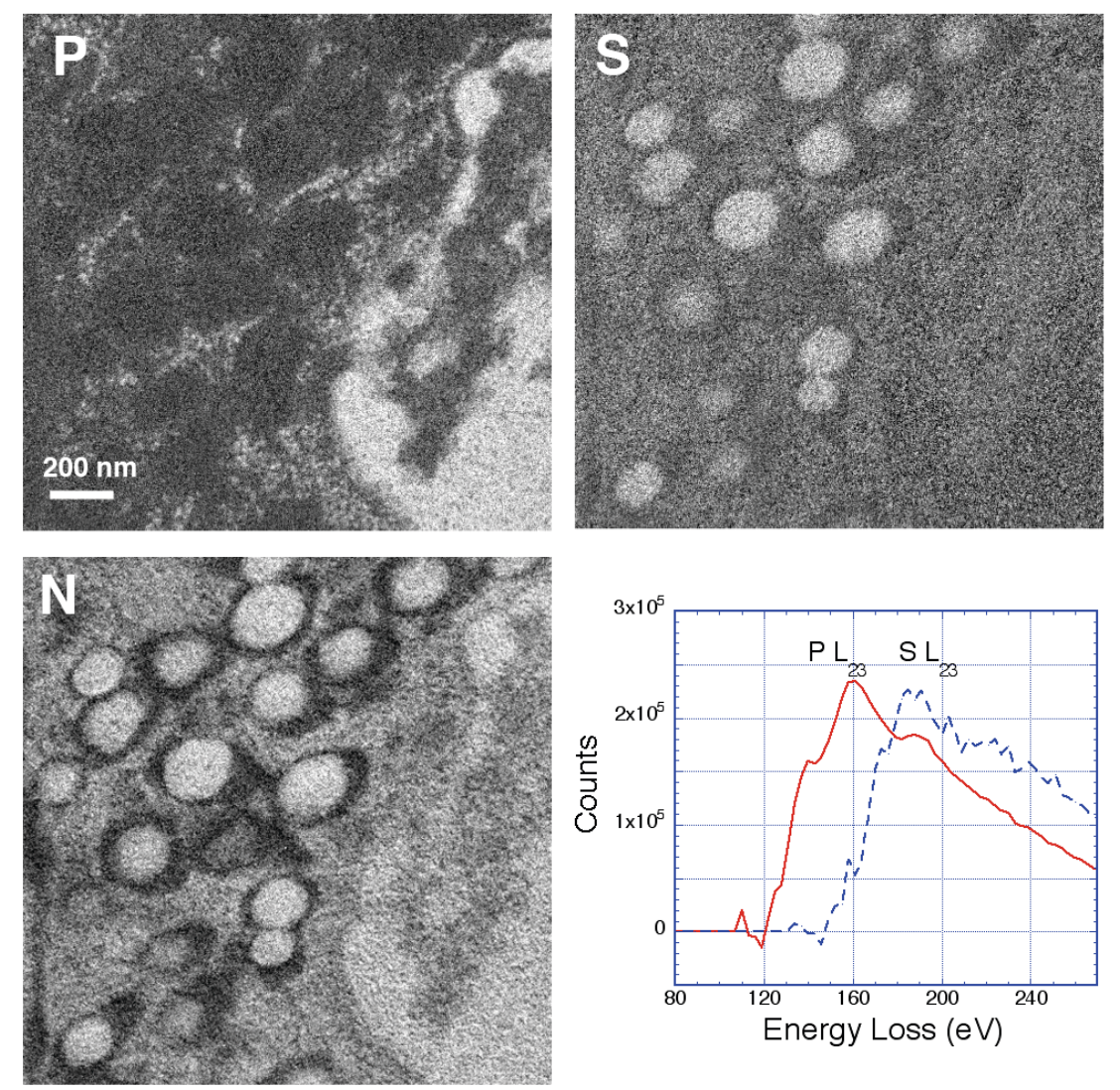

Fig. 1. EFTEM spectrum-imaging of unstained pancreatic beta cell in mouse islet of Langerhans showing phosphorus $\mathrm{L}_{2,3}$-edge $(\mathrm{P})$, sulfur $\mathrm{L}_{2,3}$-edge $(\mathrm{S})$, and nitrogen $\mathrm{K}$-edge $(\mathrm{N})$ maps. Distributions of phosphorus and sulfur were obtained by fitting the overlapping $\mathrm{P} \mathrm{L}_{2,3}$ and $\mathrm{S} \mathrm{L}_{2,3}$ EELS core-edges spectra (shown in bottom right), together with single-scattering and double-scattering background reference spectra (not shown). Elemental maps were acquired with a $10-\mathrm{eV}$ slit width and $3-\mathrm{eV}$ energy increment using a FEI Tecnai TF30 electron microscope equipped with a Gatan Tridiem Imaging Filter. Specimen was prepared by conventional fixation with glutaraldehyde, dehydration, embedding in epon and sectioning to a thickness of approximately $70 \mathrm{~nm}$. High sulfur in the secretory granules is due to sulfur-rich insulin; high phosphorus is evident in the nucleic acid of the nuclear chromatin as well as in cytoplasmic ribosomes; nitrogen is present throughout the cell in both nucleic acid and protein. 